Revue d'histoire de l'enfance « irrégulière »

Le Temps de l'histoire

1 | 1998

La protection de l'enfance : regards

\title{
Le statut de 1956 des éducateurs de l'Éducation surveillée à la lecture des publications syndicales (SNPES)
}

Marc Brzegowy

\section{(2) OpenEdition \\ Journals}

Édition électronique

URL : http://journals.openedition.org/rhei/13

DOI : $10.4000 /$ rhei. 13

ISBN : 978-2-7535-1638-0

ISSN : 1777-540X

Éditeur

Presses universitaires de Rennes

Édition imprimée

Date de publication : 15 novembre 1998

Pagination : 67-82

ISSN : 1287-2431

Référence électronique

Marc Brzegowy, "Le statut de 1956 des éducateurs de l'Éducation surveillée à la lecture des publications syndicales (SNPES) », Revue d'histoire de l'enfance « irrégulière » [En ligne], 1 | 1998, mis en ligne le 20 juin 2007, consulté le 04 décembre 2020. URL : http://journals.openedition.org/rhei/13 DOI : https://doi.org/10.4000/rhei.13 


\section{Le statut de 1956 des éducateurs de I'Éducation surveillée à la lecture des publications syndicales (SNPES)"}

À partir de 1946, dans les nouveaux établissements de l'Éducation surveillée, les IPES ${ }^{(3)}$ et les COPES, ${ }^{(4)}$ si l'on excepte les employés de l'Administration pénitentiaire maintenus provisoirement dans des fonctions d'agents de service, le personnel peut être réparti approximativement en deux catégories :

- les personnels de l'Administration pénitentiaire affectés définitivement à l'Éducation surveillée suite à une commission de reclassement de novembre 1945. On trouve parmi eux des cadres administratifs, des moniteurs et moniteurs-éducateurs recrutés lors des réformes de 1938 et pendant la période de la guerre. Le nouveau statut du 10 avril 1945 leur permet souvent d'accéder à des fonctions supérieures à celles qu'ils occupaient. Leur souci principal est de consolider leur situation; ils chercheront souvent à diriger ou influencer les nouvelles sections syndicales qui se créent.

- de nouveaux recrutés en référence au statut du 10 avril 1945 (à ce sujet voir l'article de J. Bourquin dans ce numéro), auxquels il faut ajouter quelques instituteurs détachés de l'Éducation nationale qui arrivent souvent avec une expérience du syndicalisme. Ces derniers influeront beaucoup pour que le syndicat d'Éducation surveillée adhère à la FEN ${ }^{(5)}$ et rompe les derniers liens avec les syndicats pénitentiaires.

Fin 1946-début 1947, se tiennent à Paris, dans deux établissements provisoires (rue de Crimée et rue de Madrid), deux réunions auxquelles participent des délégués plus ou moins mandatés, qui décident la création du Syndicat national de l'Éducation surveillée. Le SNPES vient de naitre et progresse rapidement : en 1956, il comptait 400 adhérents répartis sur 13 établissements.

\section{Marc \\ Brzegowy ${ }^{(2)}$}

(1) Ce texte est la communication de l'auteur aux Journées d'études L'Éducation surveillée aurait eu 50 ans, Vaucresson, les 9 et 10 novembre 1995.

(2) Directeur au CNFE-PJJ de Vaucresson.

(3) Institutions publiques d'Éducation surveillée.

(4) Centre d'observation public de l'Éducation surveillée.

(5) Fédération de l'Éducation nationale. 
Le premier congrès se réunit en 1948 à l'IPES de Saint-Hilaire. Il examina en détail les statuts du syndicat et en fit un syndicat à la fois unitaire et multicatégoriel, par opposition à la situation de division qu'avaient connu les syndicats pénitentiaires.

Contrairement à ce que l'on pourrait croire, les premières luttes syndicales amenèrent progressivement une amélioration sensible des conditions de travail ainsi qu'une revalorisation de la fonction éducative.

La lutte concernant le nouveau statut du personnel d'éducation commence par la volonté de supprimer le grade des éducateurs adjoints, qui étaient bloqués rapidement à la 4ème classe de leur grade, à l'indice 277.

Elle aboutit tout d'abord à la création d'un plus grand nombre de postes d'éducateurs, puis en 1956, au nouveau statut du personnel d'éducation, qui pour l'époque peut être cité en exemple. Dans l'étude et la mise au point des mesures transitoires, l'action du SNPES fut prépondérante.

Dès 1951, le SNPES se dote d'un outil de communication sous la forme d'un bulletin, qui paraîtra à peu près régulièrement de manière bimensuelle, et dont le premier numéro, sorti en septembre-octobre 1951, trace les premières perspectives d'action après le congrès de Belle-Ile :

"Il s'agit entre autres d'obtenir pour chacun et pour tous :

- la réalisation d'un statut définitif, d'un statut particulier supprimant les postes d'éducateurs adjoints et accordant après l'année de stage une nomination à un échelon de traitement égal au point indiciaire 220;

- l'étude d'une revalorisation ultérieure de la fonction éducative dans un cadre indiciaire allant de 225 à 410. "

Le numéro 2, paru en novembre-décembre 1951, rend compte d'une audience à l'Administration centrale en ces termes :

"Le directeur pense lui aussi qu'il faudra examiner les possibilités d'une modification des indices dès que le climat sera plus favorable; en attendant, il faudra obtenir une amélioration de l'indemnité de risque et un départ meilleur dès la fin de stage."

Dans le même numéro, un article, consacré aux délégués permanents à la Liberté surveillée, agents contractuels recrutés sur titres, rappelle leur mission, leurs fonctions et leurs conditions de recrutement : 
"L'arrêté du 20 octobre 1951 exige l'une des qualités suivantes :

- posséder un diplôme d'enseignement supérieur (licence ou équivalent);

- posséder brevet supérieur ou baccalauréat, et avoir été pendant un an délégué bénévole, assistante sociale ou éducateur spécialisé ; ${ }^{(6)}$

- avoir exercé pendant deux ans et en y donnant satisfaction, des fonctions d'éducation dans les services extérieurs de l'Éducation surveillée. "

[...] « Malheureusement, les candidats venant des services extérieurs postulant à un emploi de délégué permanent doivent abandonner leur qualité de fonctionnaire et devenir agents contractuels, ce qui, sur le plan administratif, est un non-sens. Il serait pourtant si simple, sans fonctionnariser entièrement la profession, de décider que les personnels des services extérieurs postulant pour un emploi de délégué permanent à la Liberté surveillée conserveraient leur qualité de fonctionnaire, quitte à ajouter qu'ils cesseraient de concourir pour l'avancement normal dans le cadre des établissements. "

[...] « Cette mise au point est nécessaire si on veut obtenir le personnel ayant l'expérience des mineurs délinquants et les qualités voulues pour mener à bien une tâche aussi délicate. Il n'est d'ailleurs que justice de faire en ce domaine une place favorisée aux membres des services extérieurs ayant donné dans les établissements le meilleur d'eux-mêmes et prêts à se lancer dans cette voie avec une ardeur nouvelle.

C'est la seule façon d'éviter que les postes de délégués soient accaparés par des gens qui ne cherchent avant tout qu'un "fromage" et pour qui les questions de protection de l'enfance ne sont qu'accessoires.

L'expérience qui commence n'a pas le droit d'échouer. "

En février 1952 (bulletin numéro 3), le secrétaire général fait le point sur les questions à l'ordre du jour; concernant le statut des personnels éducateurs et techniques, il précise :

«Le BN ${ }^{(7)}$ doit demander l'étude par le CTP(8) d'un statut nouveau conforme à la loi de 1946. Le but étant d'obtenir la suppression du grade d'éducateur adjoint et un échelonnement définitif, je dois confirmer la demande effectuée lors de l'audience du 13 novembre, mais il me faut pour cela votre accord.

Pour me permettre de vous donner une opinion sur la question, le 15 janvier, j'ai adressé une lettre à Lavergne, notre secrétaire fédéral, en lui demandant quelques indications sur le statut des instituteurs actuellement en cours de discussion. Éléments demandés : conditions de recrutement, nombre de classes et échelons, conditions 
(9) La Commission administrative paritaire. d'avancement, échelonnement indiciaire, horaires et congés, dispositions communes. " Un peu plus loin, un membre titulaire de la $\mathrm{CAP}^{(9)}$ s'adresse " aux camarades éducateurs adjoints ":

"Croyez-moi, camarades éducateurs adjoints, lorsqu'on voit votre qualification professionnelle et vos services rendus à la cause de l'enfance inadaptée, il est pénible pour un représentant du personnel de vous refuser le titre d'éducateurs pour le révoltant motif qu'il n'y a pas de postes. D'où la conclusion : ou bien il faut augmenter le nombre de ces postes, ou bien il faut supprimer le grade d'éducateur adjoint; pour moi, jopte pour cette dernière solution. "

Dans le même numéro, le secrétaire de la section de Saint-Jodard signe un article intitulé "Éducateurs et instructeurs "; il y dénonce les heurts qui se créent régulièrement entre éducateurs et instructeurs, et note :

"Les conditions de recrutement pour le personnel éducatif devenant de plus en plus rigoureuses, il doit en être de même pour le personnel technique, car quiconque est loyal doit reconnaître que le niveau actuellement exigé pour l'admission d'un instructeur ne correspond pas toujours aux indices 220-390.

N'est pas syndicaliste l'éducateur qui regarde l'instructeur avec ses yeux de bachelier semi-intellectuel. N'est pas syndicaliste l'instructeur qui voit dans l'éducateur un pauvre bachelier, ou un raté. Je dénonce ces attitudes parce qu'elles existent. Mesquineries, étroitesse d'esprit! Alors que nous pourrions nous compléter si richement : les uns avec leur expérience de l'usine, les autres avec leurs acquisitions intellectuelles.

Je demande aussi aux instructeurs de regarder de près le service des éducateurs, ce service en trois temps : antisocial et antifamilial, ce service du dimanche et des jours fériés : camarade instructeur père de famille, envies-tu l'éducateur qui passe le jour de Noël à l'IPES, en laissant seuls chez lui sa femme et ses enfants?"

En avril 1952, un titre vengeur barre la une du bulletin numéro 4 : "Éducateurs! Il nous faut d'autres indices ".

L'auteur, un membre du Bureau national, argumente :

"Si nous avons pu, à une certaine époque, nous estimer satisfaits d'avoir fait reconnaître la valeur de notre personnel, je dirai même de l'avoir fait admettre au sein de la fonction publique, et d'avoir obtenu l'assimilation des éducateurs aux instituteurs, grâce à notre adbésion syndicale à la FEN, il semble bien qu'il ne faille pas stationner après ce premier pas. " 
[...] Je pense qu'il nous faut faire admettre que notre fonction est une fonction entièrement nouvelle. Elle n'existait pas avant la guerre et elle ne peut être assimilée à aucune autre. Les arguments sont assez faciles à trouver, vous les connaissez, et je n'ai pas ici à vous convaincre de leur valeur ni de leur vérité.

En ce qui concerne les diplômes, je veux bien admettre avec tout adversaire que nous n'avons que le baccalauréat ou le brevet supérieur. Mais si nous n'avons rien d'autre, c'est qu'il n'y a pas en France de diplôme correspondant à nos multiples attributions. Le diplôme d'éducateur existe-t-il? Et pourtant, peut-on maintenant encore en nier la nécessité? Il nous faut un diplôme pour sanctionner l'acquisition des connaissances qu'on nous demande dans les disciplines les plus diverses (enseignement scolaire, médecine, éducation physique, droit, activités dirigées, psychologie...). Parce que ce diplôme n'existe pas, devons nous en supporter les conséquences?

D'autre part, la succession des tableaux d'avancement qu'il fut nécessaire de franchir par exemple à un sous-directeur, n'équivaut-elle pas à un ou plusieurs certificats de licence?

C'est en partant de ces bases de discussion : impossibilité d'assimilation rationnelle à une fonction déjà existante et absence de diplômes adéquats, que l'on devrait, à mon avis, soumettre et défendre nos propositions de modifications indiciaires. "

[...] «J'attends maintenant vos avis pour établir, en accord avec les autres membres $d u B N$, un projet en forme et répondant à vos désirs, à présenter aux organismes compétents. »

Le rapport d'activité présenté au congrès de Saint-Jodard d'octobre 1952, publié dans le numéro 6, en août 1952, rappelle que la question de la revalorisation de la fonction est actuellement à l'étude, et que le camarade $\mathrm{B}$ en fera le point avec les sections.

"Il faut, en effet, mettre sur pied une proposition de reclassement indiciaire propre aux fonctions de l'Éducation surveillée.

Lintervention syndicale a néanmoins abouti à la publication des textes allouant une indemnité aux éducateurs chefs et octroyant une amélioration sensible de l'indemnité de risques. Les démarches sont actuellement en cours pour que celle-ci soit étendue aux veilleurs de nuit. "

Pour mémoire et pour resituer ces préoccupations dans leur contexte, nous avons relevé les propos de M. Guy Petit, secrétaire d'État à la Fonc- 
(10) André Bernard, membre du Bureau national du SNPES en 1952. tion publique, qui a, dans un discours prononcé le 3 août, assuré que le gouvernement, dès la rentrée, présenterait ses projets de réforme fiscale, de réforme administrative et de budget social de la Nation. Il a affirmé que les fonctionnaires devraient consentir " des sacrifices", ce qui signifie que le statut de la fonction publique ne serait pas appliqué. Le gouvernement, a ajouté M. Guy Petit, ne tolérera pas qu'il y ait dans le pays des catégories de privilégiés.

La revalorisation de la fonction éducative est à l'ordre du jour du congrès de Saint-Jodard des 3, 4 et 5 octobre 1952, publié dans le numéro 7. A. Bernard ${ }^{(10)}$ fait un exposé de la question en regrettant que la plupart des sections ne lui aient pas, jusqu'alors, fait connaître leur position. Il dit les différentes positions possibles : argumentation nouvelle ou assimilation au 2ème degré de l'Éducation nationale (comme les techniques et les administratifs).

Finalement, c'est cette dernière position qui l'emporte et le BN est chargé d'étudier à fond la question pour la présenter à l'administration centrale dont le bureau du personnel pourrait contacter la Fonction publique et les Finances sur les possibilités actuelles d'une telle réalisation.

Mais deux mois plus tard, l'éditorial du numéro 8, intitulé «Ò் allons-nous?", fait le point sur la situation politique :

"Au crépuscule de cette année 52, chacun de nous est en droit de se demander ce qu'il adviendra demain... Un fait significatif s'impose en premier lieu : le Gouvernement de ce pays recherche tous les moyens propres à ne pas accroître les charges budgétaires et par là-même nous impose un immobilisme qui risque d'être mortel.

Tout syndicaliste digne de ce nom ne peut rester insensible aux menaces de l'heure; ces menaces s'appellent : "

- [...] « recul de la limite d'âge, contraignant ainsi les travailleurs à occuper des fonctions jusqu'à 60 ou 65 ans, au risque de faire des jeunes étudiants des chômeurs ;

- réforme de la sécurité sociale; l'on veut réduire en effet les garanties contre la maladie et en revenir à la formule des assurances sociales.

En ce qui concerne l'Éducation surveillée, cette politique a également de sérieuses répercussions, car, malgré la bonne volonté et le désir de la direction, les 
crédits mis à sa disposition sont sensiblement inférieurs...

Sur le plan du personnel, le directeur général n'a pas caché, lors de la dernière audience, l'hostilité du garde des Sceaux à toute mesure entrainant une augmentation des dépenses, qu'il s'agisse d'indemnités nouvelles ou d'effectifs de personnels. »

C'est pourtant au cours de l'année 1953 que, non sans difficulté, le SNPES en arrive à la rédaction d'un nouveau statut.

En avril, le représentant des éducatifs au BN rappelle qu'il avait été chargé par le congrès de Saint-Jodard d'étudier les possibilités d'une revalorisation de la fonction sur la base d'une "argumentation nouvelle ", afin de présenter un texte complet tenant compte au maximum des particularités de notre tâche et se refusant à toute assimilation à une autre fonction.

Or, dans les mois qui ont suivi, la situation était loin d'être éclaircie : trois projets prévoyaient des réalisations différentes, projets profondément dissemblables dans leur esprit et leur conception même. Dans ces conditions, le $\mathrm{BN}$ ne pouvait pas en faire la synthèse, ni soumettre un projet susceptible de réaliser l'accord de la majorité des éducateurs.

Il fallait attendre le prochain congrès.

Le BN des 1er, 2 et 3 juin 1953 examine les propositions statutaires du personnel éducatif issues d'un questionnaire envoyé aux sections et évoque la question avec le directeur général, lors d'une audience du 4 juin. Celui-ci en renvoie l'examen au mois de novembre, en demandant au SNPES de lui faire parvenir ses propositions en cette matière; il désire que le travail soit déjà bien avancé lorsque le projet viendra en discussion au Comité technique paritaire.

L'importante question du statut du personnel est à nouveau soulevée lors d'une audience accordée le 22 septembre : le directeur général est prêt à en discuter à nouveau et à en soumettre l'étude au CTP, mais il voudrait auparavant voir sortir le statut de l'enseignement du 1er degré afin de ne pas avoir à essuyer le premier les plâtres.

Le 9 novembre, un courrier est envoyé au directeur général :

"Un réel malaise se fait actuellement ressentir chez nos collègues $d u$ cadre éducatif, malaise risquant d'entraîner de sérieuses difficultés dans le recrutement, le départ des jeunes éducateurs adjoints récemment recrutés et le découragement des plus anciens. 
En effet, les statuts des personnels de l'Éducation nationale tardant à paraître et constatant la multiplicité des formalités nécessaires à la publication des textes statutaires et la lenteur de l'examen de ces textes, lenteur imputable aux services de la Fonction publique et des Finances, nos camarades craignent que la situation qui leur est faite se prolonge encore assez longtemps.

Aussi croyons-nous devoir attirer votre attention sur la situation des éducateurs adjoints et sur celle des sous-directeurs. »

C'est au cours du congrès de Savigny-sur-Orge, qui réunit, les 2, 3 et 4 décembre 1953, 21 délégués des sections et 6 membres du Bureau national, qu'est étudié le texte des statuts qui sera déposé à la Fonction publique. Un certain nombre de modifications et d'ajouts sont proposés; une motion est votée :

"Le recrutement du personnel éducatif se heurtant à de très nombreuses difficultés, en particulier à la pénible situation qui est faite à cette catégorie, le congrès national du SNPES attire l'attention de l'Administration centrale sur l'urgence de la suppression du grade d'éducateur adjoint, qui établit dans la carrière un barrage absolument inconcevable. »

L'année 1954 voit s'intensifier la lutte pour la suppression du grade d'éducateur adjoint : un article paru en avril dans le bulletin numéro 15 rend compte d'une audience, accordée le 15 mars, au cours de laquelle le $\mathrm{BN}$ a présenté les différentes revendications et suggestions étudiées au cours du dernier congrès de Savigny.

"Le Bureau national, conscient de sa responsabilité et soucieux de suivre et de respecter les décisions prises par le congrès souverain, est encore une fois intervenu sur la transformation des éducateurs adjoints, non sans rappeler que les délégués lui ont donné sur ce point précis un mandat impératif : toute patience ayant des limites, nos camarades nous ont confié la tâche d'obtenir la transformation des postes d'éducateurs adjoints en postes d'éducateurs pour le 30 mai prochain.

Si aucune décision n'était prise à cette date, ils envisageraient l'organisation d'un mouvement de grève...

Comment obtenir cette transformation?

L'Administration ne nous apprenait rien de nouveau, le 15 mars dernier, en nous rappelant que ceci était un problème statutaire.

Notre projet de statut définitif ne fait plus mention de ce grade. C'est ici que 
l'Administration est d'accord et, sur ce point, il n'y a plus à discuter.

Mais, bien que des échanges de vues et des contacts directs entre vos représentants et la direction de l'Éducation surveillée aient déjà été pris depuis un mois environ, en vue d'elaborer les grandes lignes de ce statut, les nombreuses formalités administratives sont loin d'être terminées; il est encore trop tôt pour fixer une date, mais de toute façon notre statut définitif ne sera guère en vigueur avant un an ou deux... »

En juin 1954, une tribune libre paraît dans le bulletin numéro 16, transmise par la section de Neufchâteau :

"Le métier d'éducateur est certainement un art très difficile à acquérir puisque beaucoup de nos camarades, après sept ans de service et parfois plus, sont encore éducateurs adjoints. Ce qui ne les empêche pas, ces adjoints chevronnés, de faire exactement le même travail et d'assumer les mêmes responsabilités que les éducateurs en titre... Eh bien disons les choses franchement! Les éducateurs adjoints commencent à se fatiguer; ils commencent aussi à se rendre compte qu'on se moque d'eux... (Si satisfaction ne nous est pas donnée), alors il n'y a plus qu'un moyen de sortir de cette impasse, c'est d'user de notre droit de grève. Et quand je dis grève, j'entends grève totale."

En novembre 1954, dans le numéro 17, paraît un article intitulé « Les pléthoriques":

"Tous les efforts $d u B N$, nous dit une récente circulaire, tendront cette année à obtenir la suppression de grade d'éducateur adjoint. Je suis sceptique quant au résultat; en effet, la carotte que constitue la nomination au grade d'éducateur est trop utile aux directions d'établissements pour faire trotter les ânes que nous sommes.

Cette question a été souvent évoquée et nous sommes sans doute tous d'accord là-dessus. Mais pourquoi s'arrêter en si bon chemin? Les éducateurs chefs vous semblent-ils indispensables?

Et d'abord quel est leur rôle?"

Suit un long développement qui aboutit à deux solutions :

"la suppression pure et simple du grade ou le maintien du titre et de l'indemnité, mais étant admis qu'ils n'entraînent pas la cessation des activités de groupe. "

L'ensemble des revendications statutaires et professionnelles du SNPES concernant la catégorie éducative se retrouvent dans la motion corpo- 
rative adoptée au congrès d'Aniane des 16, 17 et 18 décembre 1954, publiée dans le numéro 18, en février 1955 :

"Le congrès constate que l'action du SNPES au cours des années passées a donné satisfaction dans de nombreux domaines aux diverses catégories de personnel groupées en son sein;

- que malgré cette action les problèmes des éducatifs demeurent nombreux et entiers

- que le règlement de ces problèmes conditionne d'une façon impérative l'existence même de l'Éducation surveillée,

- affirme sa volonté de s'attacher à résoudre en priorité l'ensemble des problèmes intéressant cette catégorie et en particulier par ordre préférentiel.

Le congrès réclame :

- la transformation annuelle et automatique d'un certain nombre de postes d'éducateurs adjoints en postes d'éducateurs...

- le reclassement de la fonction éducative dans le cadre de la Fonction publique et dans celui de l'Éducation surveillée, par l'obtention d'une revalorisation de traitement, justifiée par l'importance et les difficultés inhérentes à la profession...

- la recherche de débouchés vers des professions en liaison avec le travail d'éducation - emplois réservés pour le personnel ayant accompli au moins dix ans de service actif en contact permanent avec les élèves.

Enfin le congrès réclame, avant la parution de nos statuts définitifs, la discussion préalable et officielle, dans le cadre de réunions du CTP compétent, du projet tel qu'il a été élaboré au cours du congrès national de Savigny en 1953. "

Il n'y aura qu'un second bulletin en 1955, paru en décembre, et présentant le rapport moral du Bureau national pour le congrès suivant qui se tiendra au Centre d'observation de Collonges-au-Mont-d'Or; en revanche ce bulletin fait référence aux 20 circulaires envoyées aux sections, dont l'avant-dernière diffusait le projet de statut tant réclamé par tous, et la dernière apprenait aux sections que le statut particulier du personnel éducatif était au Conseil d'État, dernier stade avant sa parution.

L’année 1955 aura été une phase active de négociation, puisque le BN a obtenu cinq audiences officielles à l'AC, dont une auprès du garde des Sceaux, et qu'il a amené devant le CTP central de l'ES le projet de statut éducatif (problème prioritaire fixé en congrès). 
La commission éducative du congrès de Collonges, réunie les 18, 19 et 20 janvier 1956, examine le texte du futur statut des éducateurs : le bulletin numéro 20, paru en mars 1956, en rend compte; sont examinés :

- les modes de recrutement

- l'échelonnement indiciaire

- la répartition des personnels ( $80 \%$ éducateurs, $20 \%$ chefs de service)

- la grille de concordance (conditions de reclassement).

La discussion fait apparaitre que ce sont les éducateurs adjoints les plus lésés, du fait de la non reconstitution de leur carrière : le congrès envisage des recours individuels en Conseil d'État et invite le BN à demander une audience avec représentation d'éducateurs adjoints, après établissement d'un rapport prouvant qu'ils sont largement défavorisés.

Parmi les autres questions à régler après statut, la commission éducative du congrès souhaite la mise en place d'une commission pédagogique « en donnant un sens très large à ce qualificatif. Il s'agit d'organiser le travail d'enseignement, technique et éducatif. Il est décidé de tenter un essai par correspondance, une ou deux pages du bulletin étant réservées à ce travail ».

Le 15 février 1956, un courrier est envoyé au directeur de l'ES ayant pour objet l'examen des nouvelles dispositions statutaires intéressant le personnel d'éducation et l'intégration du personnel en fonction... :

" un examen objectif des dispositions nouvelles nous procure la satisfaction de constater que la carrière future se traduira par des avantages importants pour les jeunes gens qui désormais postuleront à l'emploi d'éducateur. »

Pour autant, la liste est longue des difficultés liées à l'intégration du personnel actuellement en fonction; une audience est accordée le 17 février et des contacts sont pris avec la FEN pour tenter de dégager les moyens propres à éviter que certains personnels soient désavantagés.

En mai 1956, paraît le numéro 21, dont l'éditorial porte le titre " $L e$ problème prioritaire » : le $\mathrm{BN}$ s'y félicite d'avoir, dans sa réunion plénière du 23 avril dernier et avant même l'audience accordée le même jour (c'est le jour de la parution du statut au JO),

"[...] modifié la plate-forme revendicative de notre organisation et ainsi fait la preuve, une fois de plus, si cela était nécessaire, que notre syndicat n'est pas seu- 


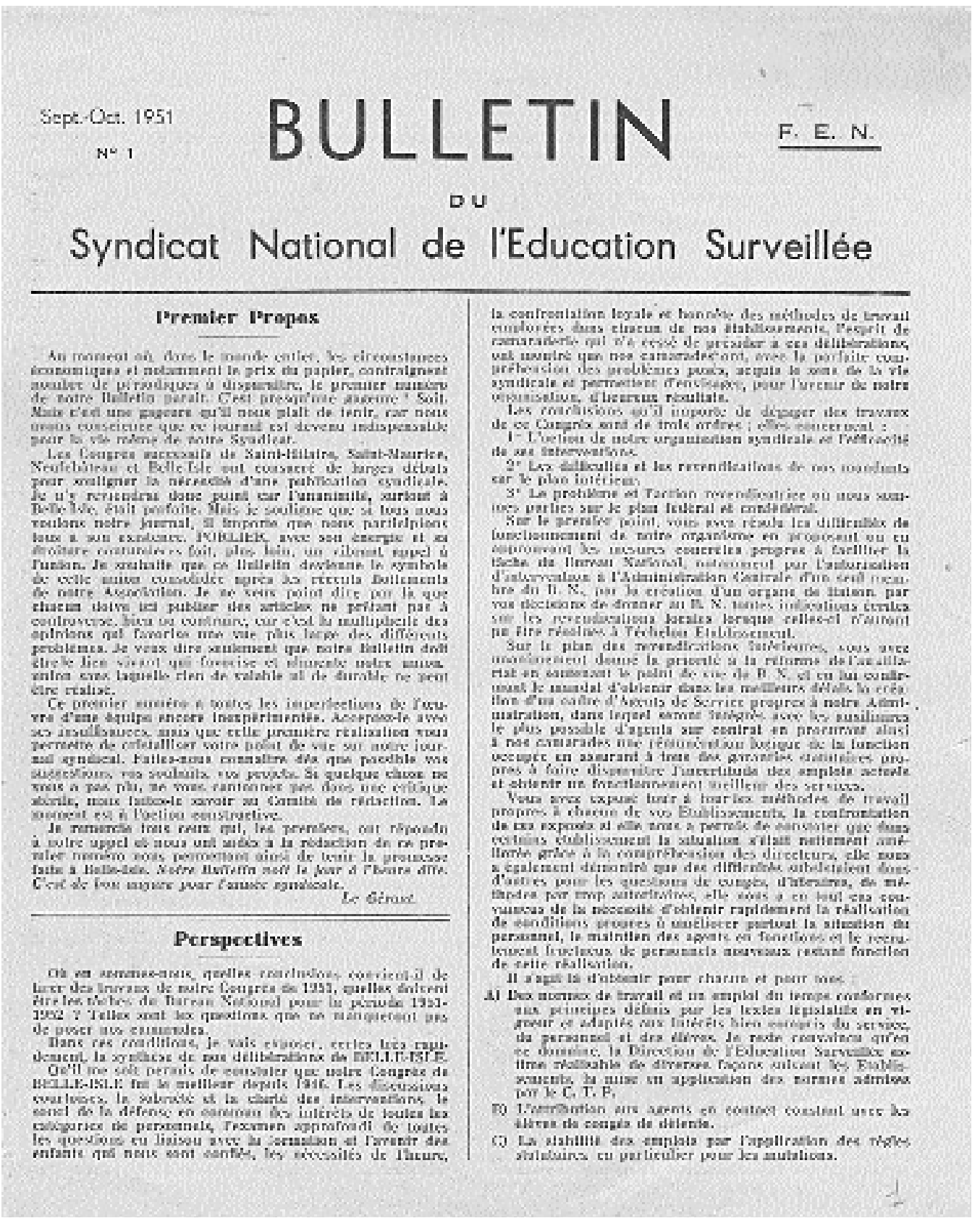

Bulletin n¹ du SNPES (1951) 


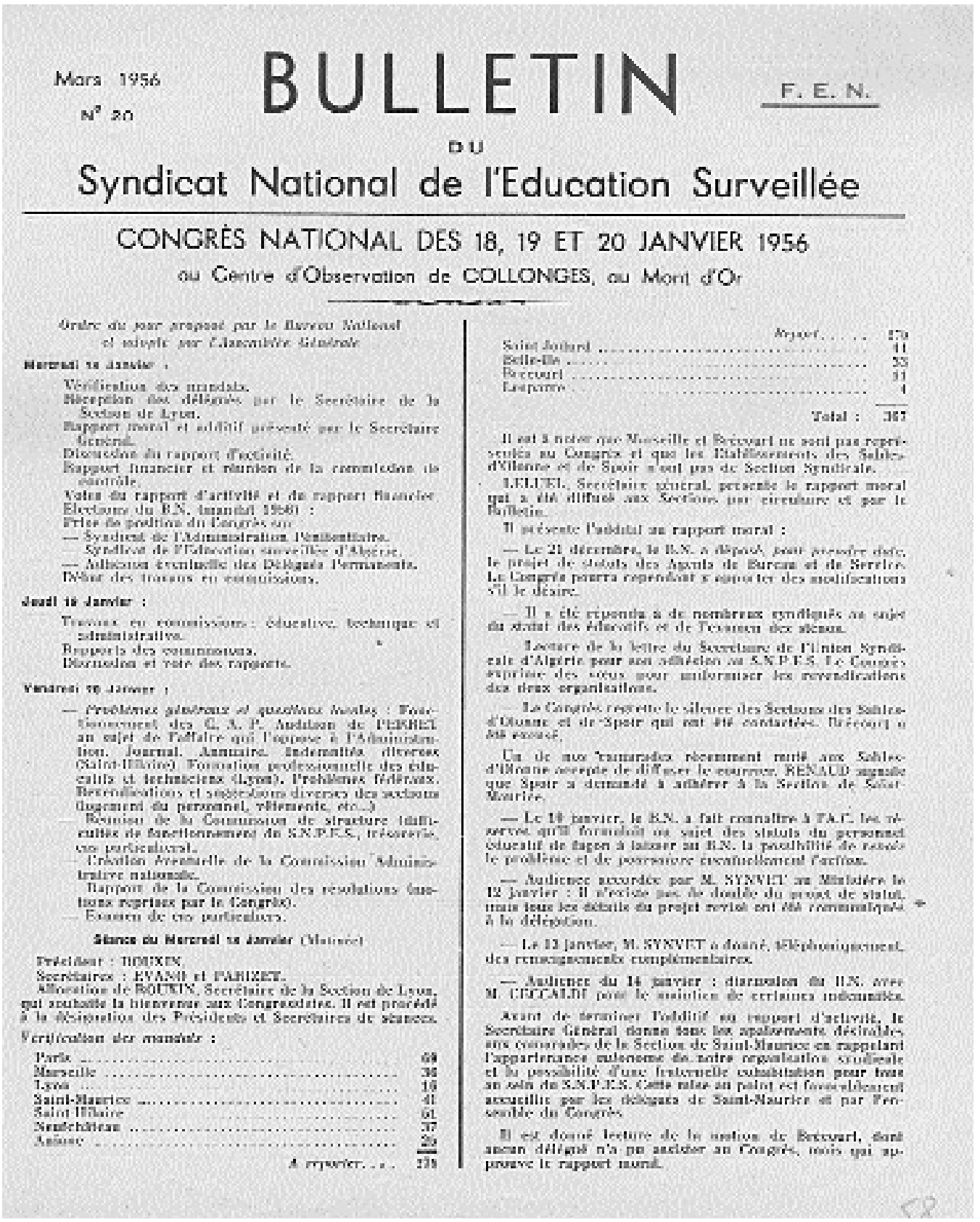

Bulletin n²0 du SNPES (1956) 
(11) Rapport annuel de la direction de l'Éducation surveillée, 1956, p. 43 .

(12) Le statut est signé par François Mitterrand, Garde des sceaux.

(13) Cette formation existe d'une manière officieuse à Vaucresson depuis 1951 . lement le syndicat des éducateurs, des techniciens ou des administratifs. Il représente effectivement toutes les catégories de nos personnels.

Le problème prioritaire pour chacun de nous à partir de maintenant est de mettre tout en auvre pour obtenir, dans les meilleurs délais, ce cadre de personnel fonctionnaire propre aux services extérieurs de l'ES que nous réclamons depuis si longtemps. "

[...] « Nous ne répéterons jamais assez que notre but numéro un est à présent de voir nos camarades des petites catégories obtenir satisfaction. "

Le statut du 23 avril 1956 opère la fusion entre les éducateurs d'internat et les délégués permanents à la liberté surveillée. Il s'agit par la même occasion, dans ce contexte de l'évolution de l'Éducation surveillée, "de rapprocher les méthodes de la rééducation en internat et en cure libre "; "la compétence de l'éducateur doit s'accroître, il pourra intervenir aussi bien dans un IPES que dans un CO, dans un service d'observation en milieu ouvert, dans un foyer, dans un service de liberté surveillée, au Centre de formation et d'études de l'Éducation surveillée ". ${ }^{(1)}$

Pour les professeurs d'éducation physique, il est prévu qu'on pourra pourvoir à leurs postes par détachement de l'Éducation nationale.

À l'inverse du statut du 10 avril 1945, celui du 23 avril $1956^{(12)}$ accorde une importance réelle à la formation. Suite à l'admission à un concours, qui nécessite le baccalauréat ou des diplômes équivalents depuis le statut de 1945, est officialisée une formation théorique et pratique de deux ans à l'issue de laquelle les éducateurs stagiaires subissent un examen professionnel en vue de leur titularisation. ${ }^{(13)}$

Dans le déroulement de carrière qui va jusqu'au grade de directeur de 1ère classe, est supprimé le premier grade d'éducateur adjoint. Le statut, qui s'inspire de celui des instituteurs, apporte une amélioration sensible à la rémunération des éducateurs, dont l'indice terminal dans ce grade passe de 360 (statut de 1945) à 430.

Dans le même numéro, un article intitulé "Tour d’horizon " fait le point sur la situation après la parution du statut :

"Le statut éducatif est paru... Les uns accueillent ces décisions avec satisfaction, les autres avec une pointe de regret. Tous cependant nous reconnaissons que la nouvelle carrière offre dans son ensemble d'incontestables avantages par rap- 
port à l'ancienne, entre autre la disparition de l'espèce "éducateur adjoint" et l'élévation du plafond indiciaire à $400 \ldots$

Sans doute la situation indiciaire est et demeure un élément important de recrutement. Je voudrais cependant relever deux autres éléments dont l'importance me paraît également primordiale. Je veux parler de la situation matérielle qui s'attache actuellement à la fonction et des conditions de travail offertes aux candidats, dont il ne faut tout de même pas attendre une ferveur et une abnégation sans réserve...

Nous attendions beaucoup à ce sujet du statut et de la dizaine d'arrêtés d'application qui doit suivre. La "généralité" des termes du statut ne nous satisfait point et les arrêtés d'application ne correspondent pas davantage à ce que beaucoup d'entre nous escomptaient y trouver...

Il reste un gros travail d'organisation à prévoir. Notre syndicat se veut de n'être pas un organisme uniquement revendicatif; nous nous devons de construire, tout au moins de participer positivement à une tâche dont nous sentons impérieusement la nécessité et qui ne saurait se passer de notre expérience.

Nous prétendons qu'au contact des réalités nous sommes à même de juger mieux que quiconque des difficultés de la profession. Les théories s'échafaudent sur des faits. Ces faits, nous les vivons quotidiennement. Nous ressentons vivement, intimement persuadés de la valeur de l'œuvre à laquelle nous coopérons, la nécessité de dégager des horaires, des programmes, des méthodes. Nous souffrons d'un manque patent d'organisation...

Nous avons maintenant un statut correct. Mais il reste encore beaucoup à faire pour améliorer la situation matérielle du personnel et presque tout faire pour organiser notre profession qui, avec ses sujétions nombreuses mais inéluctables, réclame patience et dévouement..."

Pour conclure, laissons la parole à l'auteur d'une tribune libre parue dans le numéro 25, en septembre 1957, et intitulée "Remarques sur le fonctionnement de la Commission pédagogique » :

"Je suis intimement persuadé que la réforme des institutions d'Éducation surveillée n'a pas eu lieu tout à fait en 1945, mais qu'elle se joue ces années-ci et qu'elle dépend beaucoup, quoi qu'on en pense en certaines sphères, de la manière dont nous, Éducateurs publics, saurons passer de l'ère du pionnier à celle des règles de travail normalisées, sans tomber dans l'erreur irréparable d'une "fonc- 
tionnarisation" de l'action et de la pensée, sans vivre à la remorque ou en marge des travailleurs sociaux de bonne foi qui œuvrent pour un même progrès...

Bureau d'études, la Commission pédagogique doit permettre au SNPES de devenir la pierre angulaire du corps des éducateurs, étant son organe le plus dynamique, celui qui permet immédiatement le contact avec les Associations qui poursuivent les mêmes buts.

Par ailleurs, elle n'a pas le droit de s'occuper à la légère de n'importe quoi, de s'encombrer, de se dévaluer dès sa naissance au moment où le statut des Éducateurs publics venant d'être appliqué, le recrutement se fait en majorité par la voie exceptionnelle du contrat, au moment surtout où se dessine un statut national et une définition de l'Éducateur de jeunes inadaptés. "

Il est fait là référence aux accords entre l'UNAR (Union nationale des associations régionales de sauvegarde) et l'ANEJI (Association nationale des éducateurs de jeunes inadaptés), qui seront signés en mars 1958 et qui préfigurent l'organisation et la formation des éducateurs des jeunes inadaptés, dont la convention collective interviendra en 1966.

Ces accords UNAR-ANEJI se sont souvent référés dans leurs travaux au statut des éducateurs de l'Éducation surveillée du 23 avril 1956. 